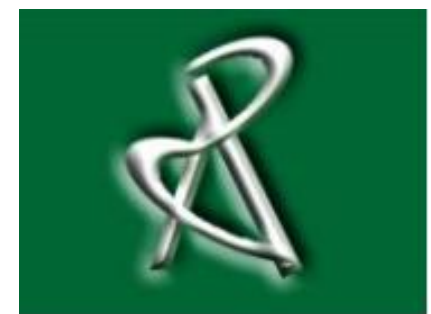

Available online at www.academicpaper.org

Academic @ Paper

ISSN 2146-9067

International Journal of Automotive

Engineering and Technologies

Vol. 3, Issue 1, pp. 32 - 39, 2014

\section{Technical Note}

International Journal of Automotive

Engineering and Technologies

http//www.academicpaper.org index.php/IJAET

\title{
Electromagnetic compatibility - electrostatic discharge tests for automotive Tamilarasu.S ${ }^{1}$, P.Thirunavukkarasu ${ }^{2}$, N.Sethupathi ${ }^{3}$ \\ ${ }^{1}$ Research Scholar, Bharathiar University, Coimbatore, India \\ ${ }^{2}$ Dept of Electronics, SRMV College, Coimbatore, India \\ ${ }^{3}$ Dept of Physics, A.A.Govt Arts College, Namakkal, India. \\ Received 15 July 2013; Accepted 03 December 2013
}

\begin{abstract}
The international automotive ESD standard (ISO 10605: 2008) has significant differences from the commercial ESD testing standard (IEC 61000-4-2). Differences include the Human Body Model (HBM) used to simulate a person's electrical network and the utilization of significantly higher discharge voltages, up to $\pm 25 \mathrm{kV}$ air discharge. This article aims to examine the differences and consider why they exist. The test methods will be explained and the similarities between the automotive and commercial product standards also highlighted. A discussion will also be forwarded on why other non-automotive products might be improved if they were designed to meet the automotive ESD standard and why this standard might be more appropriate in some non-automotive situations than IEC 61000-4-2.
\end{abstract}

Key words: Electromagnetic Compatibility, ESD, Vehicle level testing, EAS Testing

* Corresponding author

E-mail address: kongutamil@ rediffmail.com

\section{Introduction}

Our current vehicles contain three centuries 
of technology. $19^{\text {th }}$ century internal combustion engines combined with $20^{\text {th }}$ century electrical systems and $21^{\text {st }}$ century electronics. Current electronic systems (including vehicles) contain many more active electronic components than in the past. Those components and assemblies may emit RF noise or be exposed to external sources of energy -resulting in unanticipated changes in system operation. Vehicle Original Equipment Manufacturer (OEM) practices is to address EMC in the component and system design phase. Most OEM requirements are based upon international standards such as CISPR, ISO, and SAE.

\section{Automotive EMC}

Electromagnetic compatibility testing is used to identify check the electronics system sensitivities and robustness in varies conditions. Out of many tests, one important test is "Electrostatic Discharge". In this test we will simulate natural and human-body induced high voltage $(4-25 \mathrm{kV})$ discharges. This could cause immediate failure or induce latent defect. The automotive ESD standard (ISO 10605: 2008) is based on similar models and premises as the commercial standard, essentially derived from IEC 801-2:1991. The test simulates the effect of a human body discharge of static electricity, a relatively common occurrence experienced by most vehicle users, usually when entering or exiting the vehicle. The ISO 10605 standard includes testing on vehicle as well as testing of individual ESAs (Electronic Sub Assemblies). The standard was widely used by OEMs prior to its full adoption by ISO and included within many OEMs EMC specifications, including the unpowered test before this was included in this ISO standard.

\section{The Human Body Model}

The human body model (HBM) consists of a single capacitor that is charged up to the required test voltage, and then discharged through a series resistor to the ESA under test, simulating the charge accumulation on a person then discharge to the ESA/vehicle.

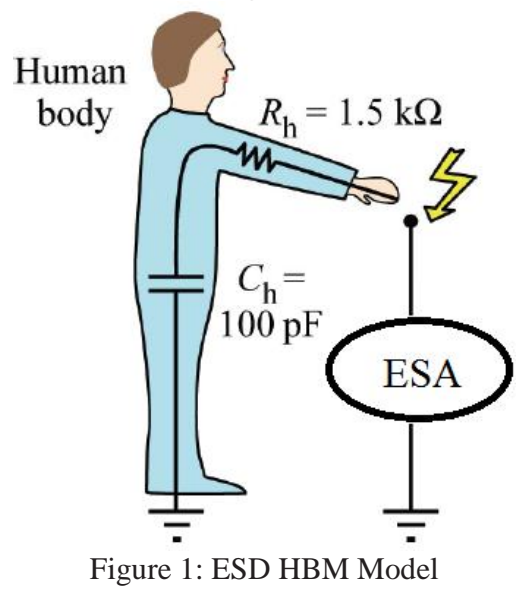

Although the HBM premise and component types are the same as commercial standards, the HBM component values are different for the automotive test, in particular the resistance is typically several hundred Ohms in commercial test standards $(330 \Omega)$ rather than $2 \mathrm{k} \Omega$ used for vehicle test. At first, many observers coming from the commercial field have difficulty understanding why the HBM has different values. The commercial standard is derived for products that essentially have a reference that can connect directly or indirectly to the ground/earth reference that the body has been charged in reference to.

However, in an automotive application, the vehicle and/or ESA during handling is in fact isolated from the earth reference. Hence, the discharge is capacitor (human) to capacitor (ESA or vehicle). Consequently, not only does it make sense for the HBM to be different for the automotive environment. It could even be argued that any equipment that is powered solely by battery power should also be tested to this automotive ESD standard rather than the commercial IEC 61000-4-2. Some commentators believe the higher resistance gives the impression that automotive ESD is an easier standard to meet than commercial ESD. The discharge voltages are higher (up to $\pm 25 \mathrm{kV}$ ); hence the actual energy impact is similar. Most automotive ESAs tend to have a higher 
immunity to ESD in practice than many commercial equivalents when tested under the same discharge and HBM conditions.

\section{Test Equipment}

The test equipment for ESD testing is relatively simple in comparison to most EMC tests. The tests can be performed over a simple $1 \mathrm{~mm}$ thick metal ground plane, and the ESD discharge device consists of a power supply capable of generating the appropriate test voltage and a test discharge head containing the appropriate human model components with switch. The tests do not need to be conducted in a screened room, although this is occasionally the case within test laboratories as the air discharge tests can register as radiated noise on nearby sensitive receivers if not shielded. During the test, ambient temperature and humidity are the influencing parameters.

The ESD power supply should to be capable of generating $\pm 25 \mathrm{kV}$ to meet ISO 10605 requirements, although this level may not be required for many tests. The discharge head is usually changeable with loads for the two different human body models and combined with a spherical test probe for air discharge and a pointed probe for contact discharge (see Figure 2). These probes are identical to non automotive ESD test probes based on IEC 61000-4-2.
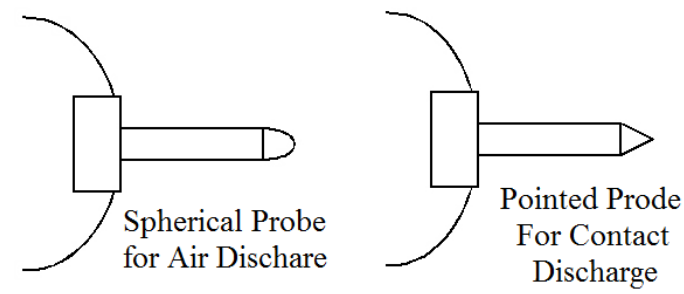

Figure 2: ESD Test Probes

\subsection{Test Pulse}

The output pulse shape from the ESD gun is not usually displayed and, it is usually in volts. But the ISO standard gives the pulse shape in amperes for calibration purposes (see Figure 3). The automotive ESD test can appear less severe than some of the commercial standard tests, not only due to using a higher resistor value in the HBM, but also a slower minimum rise time of $0.7 \mathrm{~ns}$ compared to $0.4 \mathrm{~ns}$ that can be used in contact discharge in commercial standards. Rise time for air discharge is also relatively slow at under $5 \mathrm{~ns}$. These rise times are calibrated from test contact discharges into $2 \Omega$ test loads. The slower rise time for air discharge reflects the isolation of a vehicle from the common "earth" reference. Even during transport and handling of ESAs the device is still isolated from the "earth reference." Hence, it could be argued that the automotive ESD standard is still applicable for any device that is either battery powered or handled when disconnected from its supply. As with most automotive EMC standards, there are options on the severity of discharge used.

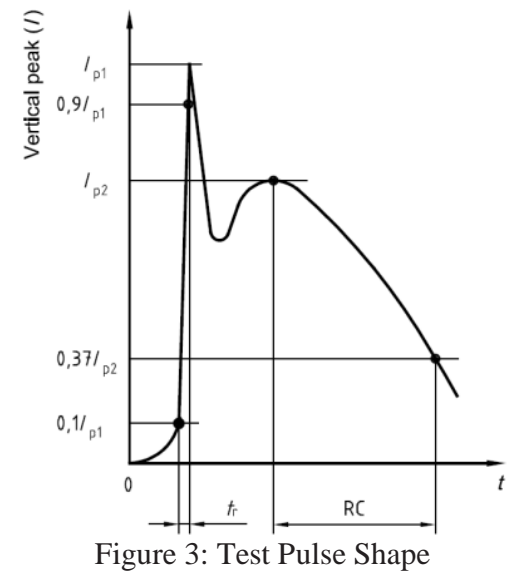

The peak currents contained in the test pulse give some indication of how severe these tests can be and the peak current levels used in automotive ESD testing are comparable to commercial ESD standards. The peak current is applied for a very short period if a discharge occurs, but this is followed by a longer discharge at lower current level after this initial peak (see Figure 3). The second discharge time is dependent on the human body model used.

\section{Test Voltage Level}

There are 4 to 6 different voltage levels that can be applied to a powered ESA, depending on the severity level and the method of discharge (air or contact). There are 4 severity classifications, labeled I to IV. But the standard contains no information on 
which to apply or how they reflect the place or function of the ESA on a vehicle. Unlike other EMC tests, the ESD test cannot be as easily classified. For example, safety critical ESAs may not have the highest severity test since these products are often the least accessible and consequently could have the lowest severity pulses applied if required.

Table 1: ESD Test Pulse Parameters

\begin{tabular}{|c|c|c|c|}
\hline Level & $\begin{array}{c}\text { Indicated } \\
\text { Voltage }(\mathrm{kV}) \\
\end{array}$ & $\begin{array}{c}\text { First Peak } \\
\text { Current (A) }\end{array}$ & $\begin{array}{c}\text { Rise Time } \\
(\mathrm{ns})\end{array}$ \\
\hline I & 2 & 7.5 & \multirow{4}{*}{$0.7-1.0$} \\
\hline II & 4 & 15 & \\
\hline III & 6 & 22.5 & \\
\hline IV & 8 & 30 & \\
\hline
\end{tabular}

Table 2: Time Constants for HBMs

\begin{tabular}{|l|l|l|l|}
\hline $\begin{array}{c}\text { Human Body } \\
\text { Model }\end{array}$ & $\mathrm{C}(\mathrm{pF})$ & $\mathrm{R}$ in $\Omega$ & $\mathrm{RC}(\mathrm{ns})$ \\
\hline Inside Vehicle & 330 & 2000 & 600 \\
\hline Outside Vehicle & 150 & 2000 & 300 \\
\hline
\end{tabular}

Unpowered tests have an additional test voltage at $\pm 25 \mathrm{kV}$ that is not used for powered ESA tests. The lowest and highest severity tests are comparable with the powered tests. It needs to be remembered that unpowered units have no direct return path for the static charge. Often the higher voltage levels do not cause a problem, as the ESA may be uniformly charged and the differential potential across individual components can remain low. On vehicle tests have 5 test voltage levels in 4 severity classifications, encompassing the full voltage range available from the ESD test gun. There is also a "manufacturer specified" option for the test severity level for all test types, where essentially any level the manufacturer requests are applied to the ESA or vehicle.

\section{Test Time}

The minimum number of pulses is specified as 3 for all tests, but it is not unusual to attempt between 5 and 10 per test point. The minimum number of pulses applies to both polarities and to each test point, rather than each test level. Hence, if say 10 test points are determined for a single severity level, then at least 60 pulses should be applied ( 3 discharges of each polarity, 10 test points). Although ESD testing is generally quicker than most EMC testing, it is a manual test technique for the most part and can be quite laborious with a large number of discharges specified at each test point. There is a minimum delay of 3 to 5 seconds between pulses, so with a large number of test points or discharges the test time can increase significantly. Increasing the delay between pulses can allow any static build up to discharge via leakage paths; however, 5 seconds is usually long enough and increasing the delay has little effect in practice. Unpowered tests require active discharging via a $1 \mathrm{M} \Omega$ bleed resistor to prevent the buildup of static due to successive discharges.

\section{Severity}

The severity levels are usually applied progressively. Hence, almost all ESAs are tested at $\pm 4 \mathrm{kV}$ up to the highest required severity level. The discharge methods are also applied progressively, air then contact where severity levels are equal. Severity for ESD tests should be applied by accessibility of the ESA. Cabin mounted, driver or passenger accessible ESAs (ICE, HVAC units) should have the highest severity as these are likely to receive most impacts, as should door modules, security products, switchgear and instrumentation. Some OEMs may classify ESAs accessible in the trunk as "outside vehicle," and use the lower capacitance test head.

\section{Functional Status}

There are 5 levels of functional status, the term concerns only the electronic functions of the ESA. The classes of functional status are common to the ISO standards for automotive EMC. Class A is the highest pass classification. As per this class A classification, device that can continue functioning, without exceeding any parametric limits, during a discharge. The lowest class E is essentially a catastrophic failure. The most common pass levels are 
class B where a device continues to work during an impact but may exceed some parameter and class $\mathrm{C}$ where a device may not function during a discharge but returns to normal operation afterwards.

The level of functional status after application of the ESD test pulse is chosen by the manufacturer; the status should reflect the expected operating classification of the ESA. The lowest status (class E) is basically a catastrophic failure and should be considered as such. ESD testing should not be catastrophic on the ESA. A suitable applied voltage level should be chosen and adhered to so that the ESA does not have to be tested to the highest available voltage level or to destruction. ESAs that are intended to be constantly accessible while the vehicle is in use should be class A functional if they are safety related or affect the control of the vehicle. Other cabin mounted equipment that does not directly affect safety or control can be class B or class $\mathrm{C}$ functional.

Under hood and non user accessible body modules only require class $\mathrm{C}$ functionality since these will not be subject to discharge during normal operation (driving). Exceptions to this last suggestion may be the engine management unit and associated ESAs that need to be class A functional during servicing.

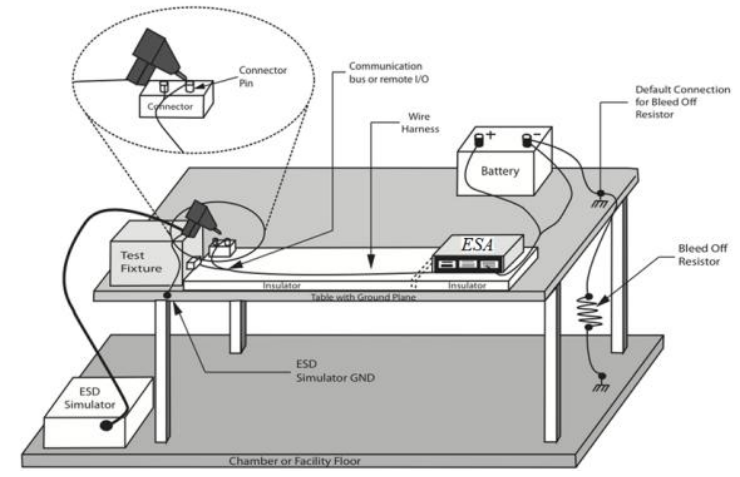

Figure 4: ESD Test Set-Up for Powered ESA Testing

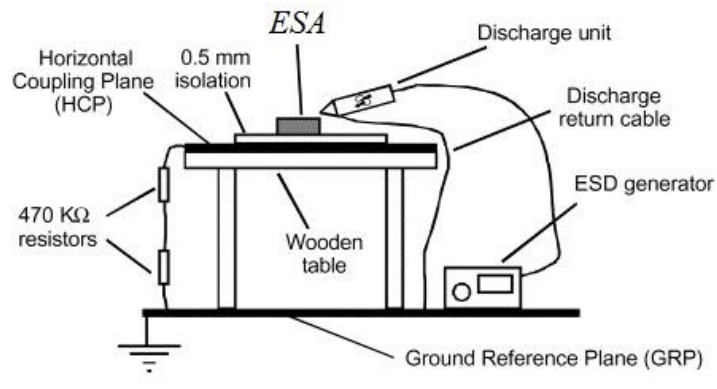

Figure 5: ESD Test Set-Up for Unpowered ESA Testing

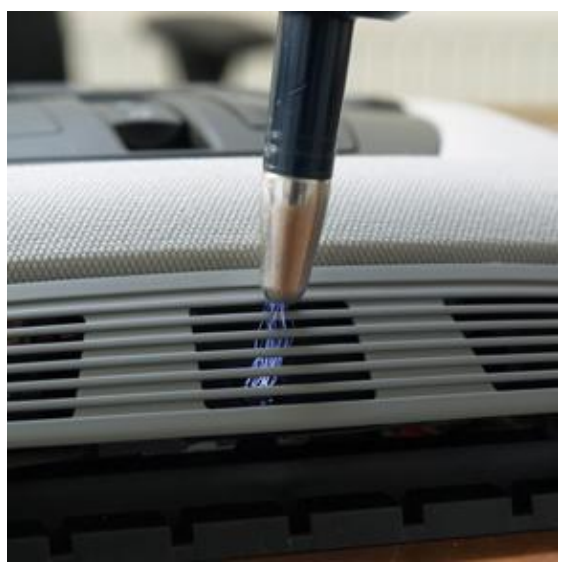

Figure 6: Testing of user accessible parts in the vehicle

\section{Testing for Automotive ESD Immunity}

Laboratory bench testing of an ESA is performed over a ground plane of at least $1 \mathrm{~m}^{2}$ and $1 \mathrm{~mm}$ thick, with over $100 \mathrm{~mm}$ from the edge of the plane to the ESA, and connected to the facility ground by a strap of less than $2 \mathrm{~m}$ in length and $50 \mathrm{~mm}$ wide. The facility grounding is made by multiple straps. The test equipment (ESD gun) is referenced to the ground plane directly. The grounding to the facility of the plane and test equipment is primarily for the safety of the test personnel rather than the ESA. A metal bodied ESA that is connected to the vehicle ground via the casing is connected directly to the test ground plane and may be placed directly on the plane for the tests. If the ESA casing is insulated from ground, such as a plastic bodied unit, the device is mounted on an insulated block $(25 \mathrm{~mm}$ high, extending $20 \mathrm{~mm}$ beyond the ESA casing on all sides). If the ESA is insulated, the connecting control lines and support equipment are also usually mounted on insulating blocks (see Figure 5). 


\section{Powered Tests}

The ESA is mounted approximately centrally on the ground plane, operating or powered and in an idle mode. Although operating during test and connected to a vehicle simulator, it is only the ESA that is tested and not its interface components or support equipment. The test is intended to test discharge during operation. Hence, the ESA connectors are not accessible to the test probe. If there are obvious places to test a discharge, a test plan is drawn up and these places only need be tested. With no test plan, the ESA should be tested across the enclosure, but there are usually more obvious points to test, since even plastic enclosures have seams and entry/exit points for cabling.

Air Discharge: The spherical probe is brought to within $15 \mathrm{~mm}$ from the ESA and the voltage applied. The tip is then moved towards the ESA until either a discharge occurs or the probe makes contact with the ESA without discharging.

Contact Discharge: The pointed probe is placed in contact with the ESA, and the discharge voltage applied.

If a discharge occurs, then the test is repeated at this voltage for the required minimum number of discharges of both polarities. If no discharge occurs, the reverse polarity is attempted. If still no discharge has occurred, then the test at that test voltage can be abandoned at that test point and a pass assumed.

The operation of the ESA is usually examined after the completion of testing at each test point. If testing for class A or class $\mathrm{B}$ functionality, the operation of the ESA has to be examined during ESD testing. This will most likely be via the support equipment, unless there is some other means of proving operation. It is particularly difficult to truly test if class A or B is achieved without some sophisticated test equipment connected to the ESA. Some of the parametric tolerances of the ESA can really only be tested with full automated test equipment and this is not often used during
ESD testing. Class A or class $\mathrm{B}$ is essentially implied from available data.

\section{Unpowered Testing}

The testing of an unpowered ESA is similar to a powered unit; the only difference is it will be without power. The support equipment is not required during the ESD test. After each successive series of impacts, and the unit is discharged to ground via a $1 \mathrm{M} \Omega$ resistor after each ESD discharge. The ESA is placed approximately centrally on the ground plane on $25 / 50 \mathrm{~mm}$ insulating blocks. The ESA has any charge dissipated after each ESD discharge via a $1 \mathrm{M} \Omega$ resistor connected to the ground plane. This can be a simple leaded resistor and is contacted to the discharge test point. Recessed pins are considered accessible during handling; if necessary, an insulated solid core wire up to $25 \mathrm{~mm}$ long can be used to access these pins. If the recessed pins are in a grounded metal connector shell, not easily accessed with a finger, then these need not be tested. The majority of automotive connectors are plastic bodied and the use of a metal probe to simulate discharge to specific pins is a valid controlled approach, even though it is sometimes considered "unfair" by some ESA suppliers. Functional testing is required before testing commences and after each test sequence. The ESA should be fully functional after the testing.

\section{On-Vehicles Testing}

Testing on vehicle is always via air discharge. The test equipment will require grounding to the vehicle, usually to the chassis/body at an exposed metal point. The test plan on vehicle includes all accessible switches, buttons within the cabin and from outside the vehicle (door locks, braking systems, lamps). The term "accessible" includes items that can be reached through open doors and windows and items accessible under hood and in the vehicle truck and/or cargo area. Externally accessed ESAs use the lower capacitance model. 


\section{Failure Mode Severity Classification}

All classifications given below are for the total device/system functional status.

The word "function" as used here concerns only the function performed by the electronic system.

Class A: All functions of a device or system perform as designed during and after exposure to interference.

Class B: All functions of a device/system perform as designed during exposure, but one or more of them may go beyond the specified tolerance. All functions return automatically to within normal limits after exposure is removed. Memory functions shall remain class A.

Class $C$ : One or more functions of a device or system do not perform as designed during exposure but return automatically to normal operation after exposure is removed.

Class D: One or more functions of a device or system do not perform as designed during exposure and do not return to normal operation until exposure is removed and the device or system is reset by a simple "operator/use" action.

Class E: One or more functions of a device or system do not perform as designed during and after exposure and cannot be returned to proper operation without repairing or replacing the device or system.

\section{Results}

Tests are normally conducted up to a specified severity, typically after- market products at levels II or III depending on location and functional classification between $\mathrm{A}$ and $\mathrm{C}$. Line fit products typically require the highest severity levels (III and IV) and often only class A or B functionality is accepted. The ISO 10605 standard includes example test tables listing functional classification for various severity levels for powered ESA and on vehicle tests. These are not particularly useful as they include the example of catastrophic failure for the highest severity level and, as already mentioned, testing should not necessarily be destructive.
ESD testing is always done progressively, so the manufacturer only needs specify the highest severity requirement and acceptable pass criteria at this severity. A better example of a test specification is simply to specify the maximum level and acceptable functional classification. The test house will make an assessment of classification of pass regardless of what the supplier specifies. The on vehicle category is rarely supplied to a test house when an ESA is submitted for testing and the powered test is intended to be equivalent. On vehicle testing is mainly performed by the VM or their test service, sometimes at a higher severity level than that to which the component was tested to in the laboratory. The vehicle itself (the bodywork and chassis) provide a degree of protection from ESD as this provides a return current path for the discharge that is likely to be much lower impedance than any wired return. Consequently, it is not untypical to have $\pm 8 \mathrm{kV}$ limit for laboratory tests and $\mathrm{a} \pm 15 \mathrm{kV}$ for on vehicle tests for the same ESA.

\section{Conclusion}

ESD testing for the automotive environment is well established and practiced in the automotive electronics industry. The test method maintains many similarities with the commercial standard it is derived from, with the main difference being the HBM change to reflect the discharge of capacitor to capacitor of two systems isolated from earth reference. It could be argued that this use of the charge transfer HBM is, in fact, more appropriate to equipment outside the automotive environment that is primarily battery powered (i.e., where there is no mains charging unit, e.g., battery operated toys) or handling tests on any product while not connected to a supply. But the probability is that this change would be more confusing to the commercial test field and I expect the test methods and HBMs will remain divergent. 


\section{Reference}

[1] CISPR-25: 2008 - Limits and methods of measurement of radio disturbance characteristics for the protection of receivers used on board vehicles.

[2] ISO 7637: Electrical disturbance by conduction and coupling Part 1: Passenger car and light commercial vehicles with nominal $12 \mathrm{~V}$ supply voltage - electrical transient conduction along supply lines only. (1990) Part 2: Commercial vehicles with nominal $24 \mathrm{~V}$ supply voltage - electrical transient conduction along supply lines only. [3] ISO 11452: Road vehicles - electrical disturbances by narrowband radiated electromagnetic energy - component test methods

[4] ISO 10605: Road vehicles - electrical disturbances from electrostatic discharges

[5] O'Hara, M., Miller, P. and Wyatt, M., Sources of Variability in Semi-Anechoic Chamber Radiated Emissions Measurement, York EMC '99, 12-13 July 1999

[6] Connor, F.R., Antennas, 2nd ed., Arnold, London, 1989.

[7] Sander, K.F. and Reed, G.A.L., Transmission and Propagation of Electromagnetic Waves, Cambridge University Press, Cambridge.

[8] EIA/JEDEC Test Method C101, "FieldInduced Charged-Device Model Test Method for Electrostatic Discharge Withstand Thresholds of Microelectronic Components," Electronic Industries Association.

[9] IEC 61000-4-2 (1995-01), "Electromagnetic Compatibility (EMC) Part 4: Testing and Measurement Techniques- Section 2: Electrostatic Discharge Immunity Test," International Electrotechnical Commission, Geneva, Switzerland, 1995.

[10] P.R. Bossard, R.G. Chemelli, and B. Unger, "ESD Damage from Triboelectrically Charged IC Pins," Electrical Overstress/Electrostatic Discharge Symposium Proceedings, ESD Association, Rome, NY. 